University of Nebraska - Lincoln

DigitalCommons@University of Nebraska - Lincoln

May 1999

\title{
Effect of dipolar molecules on carrier mobilities in photorefractive polymers
}

Arosha Goonesekera

University of Nebraska - Lincoln

Stephen Ducharme

University of Nebraska - Lincoln, sducharme1@unl.edu

Follow this and additional works at: https://digitalcommons.unl.edu/physicsducharme

Part of the Physics Commons

Goonesekera, Arosha and Ducharme, Stephen, "Effect of dipolar molecules on carrier mobilities in photorefractive polymers" (1999). Stephen Ducharme Publications. 16.

https://digitalcommons.unl.edu/physicsducharme/16

This Article is brought to you for free and open access by the Research Papers in Physics and Astronomy at DigitalCommons@University of Nebraska - Lincoln. It has been accepted for inclusion in Stephen Ducharme Publications by an authorized administrator of DigitalCommons@University of Nebraska - Lincoln. 


\title{
Effect of dipolar molecules on carrier mobilities in photorefractive polymers
}

\author{
Arosha Goonesekera ${ }^{\text {a) }}$ and Stephen Ducharme ${ }^{\text {b) }}$ \\ Department of Physics and Astronomy, Center for Material Research and Analysis, University of Nebraska, \\ Lincoln, Nebraska 68588-0111
}

(Received 14 December 1998; accepted for publication 20 January 1999)

\begin{abstract}
The grating formation speed in photorefractive polymers is greatly reduced by highly polar molecules incorporated by necessity in large concentrations to produce large diffraction efficiency and two-beam energy coupling gain. The random electric fields generated by these dipoles interfere with charge transport by increasing the width of the hopping site energy distribution and thus greatly reducing the carrier mobility and the photorefractive speed. We conducted transport studies of several model systems consisting of combinations of two polymer binders, six charge transport agents (four for holes and two for electrons), and varying concentrations of two highly polar electro-optic chromophores. The results confirm that carrier mobility is greatly reduced in the presence of polar molecules in accordance with the predictions of models of hopping transport in the presence of dipolar disorder. The randomly positioned and oriented dipoles increase the width of the hopping site energy distribution by an amount proportional to the square root of the dipole concentration and to the strength of the dipole moment. The results also show that transport agents with smaller dipole moments reduce the sensitivity to the dipolar effect. The photorefractive speed may therefore be increased by using transport agents with small dipole moments. (C) 1999 American Institute of Physics. [S0021-8979(99)00509-5]
\end{abstract}

\section{INTRODUCTION}

Charge transport is one of the key processes that control the sensitivity of hologram formation in photorefractive polymers and therefore it has become a vital consideration in the development of low cost high performance photonic devices using these materials. Since the discovery of photorefractive polymers, ${ }^{1}$ the response strength, e.g., holographic diffraction efficiency or two-beam energy coupling gain, has improved greatly. ${ }^{2-5}$ However, the speed of response, which is proportional to the photoconductivity, remains unacceptably low for many applications, with best response times in milliseconds (at $1 \mathrm{~W} / \mathrm{cm}^{2}$ optical intensity). ${ }^{6,7}$ The photorefractive speed is proportional to the charge carrier mobility and yet recent studies ${ }^{8-11}$ have revealed that carrier mobilities are greatly suppressed in the presence of dipolar species. Since chromophores with large dipole moments are necessarily present at large concentrations to provide large electrooptic response, it is essential to find means to mitigate the dipolar mobility suppression in photorefractive polymers.

The photorefractive effect is a reversible mechanism for formation of refractive index holograms in electro-optic materials. ${ }^{12}$ Photorefractive holograms have potential application in integrated optics, optical data storage, optical computing, and several other areas. ${ }^{13}$ Nonuniform illumination, for example the interference fringes of intersecting coherent optical beams, generates free carriers in the bright regions and these carriers drift and diffuse into the darker regions where they are retrapped. This spatially redistributed charge

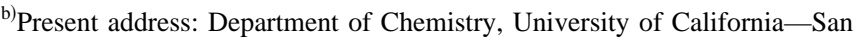
Diego, La Jolla, CA 92037.

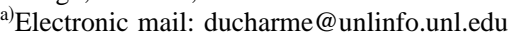

in turn generates a space charge field which changes the index of refraction through the linear electro-optic response (the Pockels effect), creating a phase replica, or hologram, of the original intensity pattern.

Since the early 1970 's, considerable progress has been made in characterizing charge transport in molecularly doped polymers (MDPs) particularly those polymers used in xerographic photoreceptors. ${ }^{14}$ Transport studies show that the carrier mobility is roughly proportional to $\rho^{2} \exp [\beta \sqrt{E}$ $\left.-\Delta_{0} / T-2 \rho / \rho_{0}\right],{ }^{15-17}$ where $\rho$ is the average separation of transport sites, $\rho_{0}$ is a constant, $E$ is the electric field, $T$ is the absolute temperature, $T_{0}$ is a constant, $\beta$ is the PooleFrenkel coefficient, and $\Delta_{0}$ is the activation energy at zero field. Transport models are mostly distinguished by the form of charge transfer between transport molecules (e.g., small polaron ${ }^{18}$ or adiabatic ${ }^{19}$ hopping), by the distributions of site energies and positions, ${ }^{20,21}$ and by the formalism for combining hopping events to calculate bulk transport. ${ }^{20,22,23}$ Recent attention has been focused on reproducing the $\exp (\beta \sqrt{E})$ Poole-Frenkel form which the current models reproduce only over a limited range of electric fields, much more limited than the available data require. ${ }^{23-25}$

Many recent results have been interpreted within the framework of the Gaussian disorder model (GDM) developed by Bässler and co-workers. ${ }^{14,20,26}$ The GDM is based on the assumption that charge transport occurs by activated hopping with a Miller-Abrahams asymmetric hopping probability ${ }^{19}$ through a manifold of localized states with superimposed energetic (diagonal) and positional (off diagonal) disorder with Gaussian distributions. Monte Carlo calculations with the GDM reproduce the Poole-Frenkel form over a limited range of fields and assume a variation of the activation energy form with $1 / T^{2}$ replacing $1 / T$. (The latter 
two forms are difficult to distinguish experimentally, given the limited temperature range of most transport measurements.) Over a limited range of electric field the GDM mobility has the form ${ }^{20}$

$$
\mu(T, E)=\mu_{0} \exp \left\{C\left[\left(\frac{\sigma}{k_{B} T}\right)^{2}-\Sigma^{2}\right] \sqrt{E}-\left(\frac{2 \sigma}{3 k_{B} T}\right)^{2}\right\},
$$

where $\sigma$ is the width of the Gaussian energy distribution of hopping states, $\Sigma$ the parameter that describes the degree of positional disorder, $\mu_{0}$ a prefactor mobility, and $k_{B}$ is the Boltzmann constant. The empirical constant $C$ has a value of $\sim 3 \times 10^{-4}(\mathrm{~cm} / \mathrm{V})^{1 / 2}$ for a wide range of MDPs. ${ }^{14}$ An increase in energy width $\sigma$ reduces the overall mobility because the (Miller-Abrahams) hopping process is exponentially activated on uphill hops; a wider range of energy differences leads to lower overall hopping probabilities. A more detailed description of the assumptions and predictions of the GDM have been given elsewhere. ${ }^{14,20,26}$ The validity of the GDM has been tested on a large number of systems, mostly by Borsenberger and collaborators (see Ref. 14 and references therein). Similar Monte Carlo calculations by Garstein and Conwell (comparing Miller-Abrahams and small-polaron hopping events), ${ }^{21,24,27}$ and by Dunlap (incorporating continuous time random walk processes) ${ }^{25}$ attempt to increase the range of the $\exp (\beta \sqrt{E})$ dependence in these models.

Recent studies of xerographic polymers show considerable evidence that the energy width $\sigma$ of the hopping site distribution can be influenced by the random electric fields of polar molecules in the material. ${ }^{28-34}$ The dipolar effect on charge transport in doped polymers has been described in a qualitative manner by a model based on dipolar disorder. ${ }^{28,30}$ The more quantitative descriptions can be found in later work, where the random potentials due to the electric fields of the dipoles are incorporated into the Gaussian disorder model, ${ }^{35-37}$ or derived from a one-dimensional random potential model. ${ }^{38}$

The main argument of the dipolar disorder model is that the local variation of the electrostatic potential resulting from randomly distributed and oriented dipoles increases the width $\sigma$ of the Gaussian energy distribution. The total energy width $\sigma$ includes the dipolar contribution $\sigma_{d}$ in addition to the usual van der Waals contribution $\sigma_{\mathrm{vdw}}$. The two contributions are assumed statistically independent and are therefore combined in quadrature ${ }^{28,35-38}$

$$
\sigma^{2}=\sigma_{d}^{2}+\sigma_{\mathrm{vdw}}^{2}
$$

The dipolar contribution $\sigma_{d}$ always increases the total energy width $\sigma$ and therefore decreases the mobility [see Eq. (1)]. The dipolar component $\sigma_{d}$ is proportional to the dipole moment $p$ of the polar molecules ${ }^{35-38}$

$$
\sigma_{d}=A \frac{c^{n} p}{\kappa a^{m}},
$$

where $\kappa$ is the dielectric constant. The quantitative models ${ }^{35-38}$ differ slightly in the value of the constant $A$ and in the dependence on the dipole concentration $c$ and the transport site separation $a$ as follows: Dieckman, Bässler, and Borsenberger ${ }^{36}$ and Hirao and Nishizawa ${ }^{37}$ find $\sigma_{d}$ $\propto c^{2 / 3} a^{-2}$; Young ${ }^{35}$ finds $\sigma_{d} \propto c^{1 / 2} a^{-2}$, and Dunlap, Parris and Kenkre ${ }^{38}$ find $\sigma_{d} \propto c^{1 / 2} a^{-1 / 2}$. The experimental evidence for the dipolar disorder models and for the form of Eqs. (2) and (3) is quite strong, ${ }^{8,9,11,29-32,37,39,40}$ but there are some studies that appear to contradict this form. ${ }^{33,41,42}$

The observation of mobility reduction by dipolar disorder in molecularly doped polymers underlines the importance of similar studies of charge transport in photorefractive polymers, which necessarily contain large concentrations of polar nonlinear chromophores. ${ }^{1,43}$ Our previous reports ${ }^{8-10,44}$ showed that the prototypical photorefractive polymer indeed had much lower mobility than expected from the type and concentration of transport agents. In this article we describe the effect of dipole moment on carrier mobility with several key components of photorefractive polymers: electro-optic chromophores, charge transport agents, and polymer binders. [Other common constituents not covered in the present study, such as sensitizers and plasticizers, may also be polar. Their effects should also follow the predictions of Eqs. (2) and (3).] A prototypical photorefractive polymer composite generally consists of a host polymer binder, an electro-optic chromophore, a photosensitizer, and a charge transport agent, where each component has a different function. The host polymer acts as the physical binder. The chromophore produces the necessary linear electro-optic (Pockels) response. The photosensitizer releases charge carriers following photoexcitation. The transport agent transfers the charge carriers though the material. These components are incorporated either as guests or covalently attached to the host polymer binder.

\section{SAMPLE PREPARATION}

For the present study we made thin films of several composites of the three key components, the host polymer binder, the electro-optic chromophore, and the transport agent, in the ratio (host):(transport agent):(chromophore) $=(45 \%-70 \%):(30 \%):(25 \%-0 \%)$ by weight. The chemical structures of these components are shown in Fig. 1 and their names and dipole moments listed in Table I. The two host polymers [Fig. 1(a)] were bisphenol- $A$ polycarbonate (PC), the backbone polymer used in the original ${ }^{1}$ and several other ${ }^{43,45-47}$ photorefractive composites and polystyrene (PS), a common host used in xerographic photoreceptors. ${ }^{14}$ The two chromophores [Fig. 1(b)] have been used before in photorefractive polymer composites: $4^{\prime}$ nitro-4'aminostilbene (NAS) (Refs. 2, 46, 48) and 2,5-dimethyl-4(p-nitrophenylazo)phenol (EHDNPB). ${ }^{49}$ The six transport agents shown in Fig. 1(c) have been studied in the context of xerographic photoreceptors. ${ }^{14}$

The solutions of charge transporting polymer composites were prepared by dissolving predetermined amounts of the host polymer, electro-optic chromophore, and charge transport agent in dichloromethane. The amounts of each component were chosen such that the dry polymer would consist of $30 \%$ by weight of transport agent, $0 \%-25 \%$ wt $\%$ of chromophore, and the remainder of the host polymer. The solids content in the solutions was between $6 \%$ and $10 \%$ by weight. 


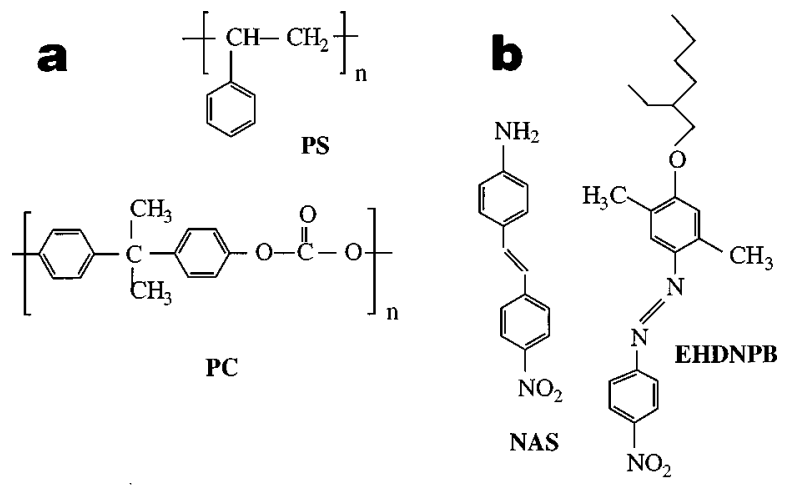<smiles>COc1ccc(N(c2ccc(OC)cc2)c2ccc(OC)cc2)cc1</smiles><smiles>Cc1ccc(N(c2ccc(C)cc2)c2ccc(C(C)(C)C)cc2)cc1</smiles><smiles>CCCCCCCC</smiles><smiles>[R]C(C)CC(C)C</smiles><smiles>[R]N1C(=O)c2ccc3c4c(ccc(c24)C1=O)N([R])C3=O</smiles><smiles></smiles>

FIG. 1. Structures of the molecules used for this study. (a) Host polymer binders: bisphenol- $A$-polycarbonate (PC) and polystyrene (PS). (b) Electrooptic chromophores: $4^{\prime}$ nitro-4' -aminostilbene (NAS) and 2,5-dimethyl-4(p-nitrophenylazo)phenol (EHDNPB). (c) Charge transport molecules: tri- $p$ tolylamine (TTA), tri- $p$-anisylamine (TAA), diethylamino-benzaldehyde diphenyl hydrazone (DEH), 1,1-bis(di-4-tolylaminophenyl)cyclohexane (TAPC), $N, N^{\prime}$-bis(1,2-dimethylpropyl)-1,4,5,8-napthalenetetracarboxylicdiimide (NTDI), 1,1' '-doxo-2-(4-methylphrnyl)-6-phenyl-4-(dicyanomethylidene)thiopyran (PTS)

The solutions were thoroughly dissolved and then filtered to $0.2 \mu \mathrm{m}$.

The samples were prepared in a multilayer thin film structure shown in Fig. 2. A polyethylene terephthalate substrate coated with a semitransparent nickel electrode $(0.03$ $\mu \mathrm{m})$ was obtained from Kodak. An amorphous-selenium $(\alpha-$ $\mathrm{Se})$ charge generation layer was deposited by vacuum evaporation on top of the nickel electrode. The thickness of the $\alpha$-Se layer was $0.3 \mu \mathrm{m}$, thick enough to absorb essentially all the $532 \mathrm{~nm}$ pump light (absorption coefficient 2.5 $\times 10^{5} \mathrm{~cm}^{-1}$ ) yet thin enough that carriers generated in the layer had a high probability of injection into the composite charge transport layer. A solution containing host polymer, chromophore, and charge transport agent dissolved in dichloromethane, was coated on top of the $\alpha$-Se layer by the "doctor-blade" technique and dried at $40{ }^{\circ} \mathrm{C}$ in argon for 4 $\mathrm{h}$. This technique consists of drawing the polymer solution
TABLE I. Acronyms, chemical names, and dipole moments of the components.

\begin{tabular}{|c|c|c|}
\hline Acronym & Name & $\begin{array}{l}\text { Dipole } \\
\text { moment } \\
\text { (D) }\end{array}$ \\
\hline \multicolumn{3}{|c|}{ Host polymers } \\
\hline $\mathrm{PC}$ & bisphenol- $A$-polycarbonate & 1.0 \\
\hline PS & polystyrene & 0.1 \\
\hline \multicolumn{3}{|c|}{ Electro-optic chromophores } \\
\hline NAS & 4 ' nitro- 4 ' -aminostilbene & 6.7 \\
\hline EHDNPB & 2,5-dimethyl-4-( $p$-nitrophenylazo)phenol & 7.6 \\
\hline \multicolumn{3}{|c|}{ Hole transport agents } \\
\hline TTA & tri- $p$-tolylamine & 0.8 \\
\hline TAPC & 1,1-bis(di-4-tolylaminophenyl)cyclohexane & 1.0 \\
\hline TAA & tri-p-anisylamine & 2.1 \\
\hline DEH & diethylamino-benzaldehyde diphenyl hydrazone & 3.2 \\
\hline \multicolumn{3}{|c|}{ Electron transport agents } \\
\hline NTDI & $\begin{array}{l}N, N^{\prime} \text {-bis(1,2-dimethylpropyl)-1,4,5,8- } \\
\text { napthalenetetracarboxylicdiimide }\end{array}$ & 3.3 \\
\hline PTS & $\begin{array}{l}\text { 1,1'-doxo-2-(4-methylphrnyl)-6-phenyl-4- } \\
\text { (dicyanomethylidene)thiopyran }\end{array}$ & 4.0 \\
\hline
\end{tabular}

across the substrate (maintained at a temperature of $35^{\circ} \mathrm{C}$ ) with a stainless-steel blade held securely a fixed distance above the film. The thickness and quality of the charge transport layer made by the above procedure depends on the blade height, solution viscosity, solid content, temperature, and draw rate. The charge transport layers for the present study were made with a $50 \mu \mathrm{m}$ blade height on a $35^{\circ} \mathrm{C}$ substrate and resulted in films $2-8 \mu \mathrm{m}$ thick, as determined from capacitance measurements. Finally, ten to fifteen circular counter electrodes of $\mathrm{Ag}$ with $0.03 \mu \mathrm{m}$ thickness and $5 \mathrm{~mm}$ in diameter were vacuum sputtered through a mask on top of the charge transport layer to complete the sample. A dielectric constant of 3 (the dielectric constant is approximately 3 for similar transporting MDPs) ${ }^{14}$ was used to determine the layer thickness and to calculate the net voltage $V$ across the transport layer. ${ }^{44}$ The sample was mounted on a copper block maintained at constant temperature stable to $\pm 0.1 \mathrm{~K}$, inside an insulated aluminum box that shielded the sample from

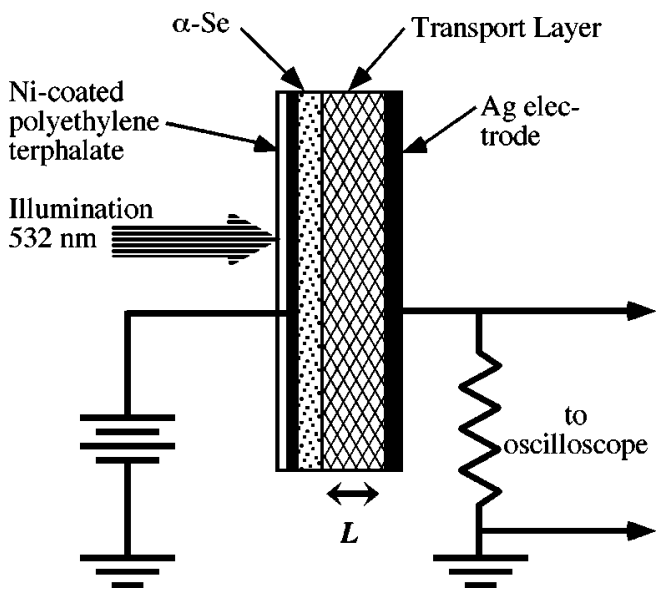

FIG. 2. Diagram of the sample and the experimental arrangement for the TOF measurements. Sample construction and measurement procedures are described in the text. 


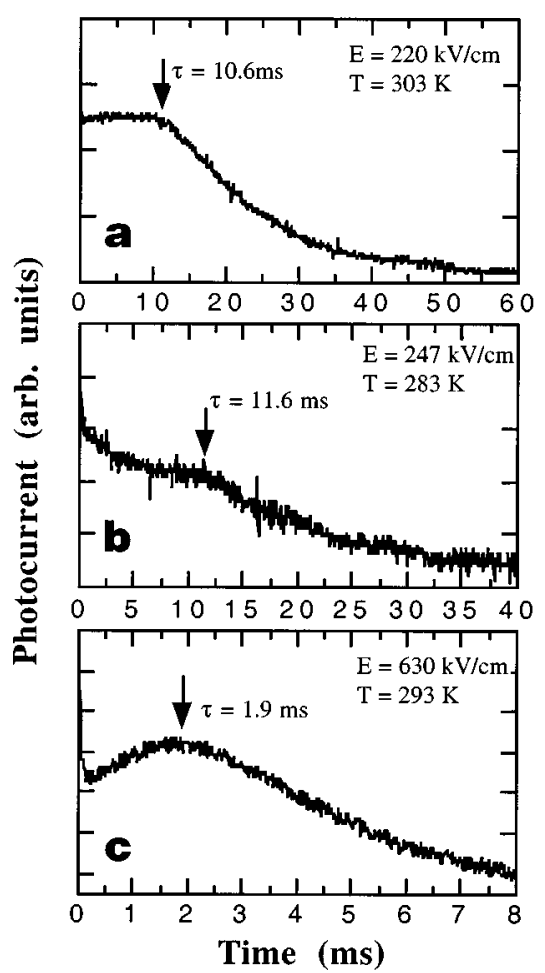

FIG. 3. Three types of photocurrent transients from the present study, (a) a clear plateau at low electric field in PC:15\%EHDNPB:30\%DEH, (b) decay followed by a plateau, signifying charge trapping in PS:20\%EHDNPE:30\%DEH, and (c) a cusp at high electric field in PC:5\%NAS:30\%DEH.

external thermal and electrical interference. The method of preparation and the geometry of the samples were described in greater detail elsewhere. .11,14,39,44

\section{EXPERIMENTAL METHODS}

The mobility measurements were made by the conventional time-of-flight (TOF) technique shown in Fig. 2. The $532 \mathrm{~nm}$ second-harmonic radiation from a pulsed Nd:YAG laser, with a pulse duration 4-6 ns, generated a sheet of carriers photoexcitation in the $\alpha$-Se layer. One of the photoinduced charges drifted under the influence of the applied electric field across the transport layer, producing a displacement current in the external circuit (shown in Fig. 2) until the charges reached the counter electrode. The doped charge transport agent and the polarity of the bias voltage determined which carriers (i.e., holes or electrons) are swept across the film. The photocurrent transients like those shown in Fig. 3 were recorded with a digital oscilloscope. The resistance $\times$ capacitance $(\mathrm{RC})$ time constant of the circuit was always much less than the transit time. During these measurements, the optical exposure was limited such that the charge injected into the polymer layer is much less than $C V$, where $C$ is the sample capacitance and $V$ is the voltage applied to the transport layer. This limitation ensures that the potential $V$ and the electric field $E$ are constant during the transient. The samples were short circuited for sufficient time after each exposure to eliminate any space charge buildup. The photocurrent transients were repeatable with no indication of hysteresis or aging.

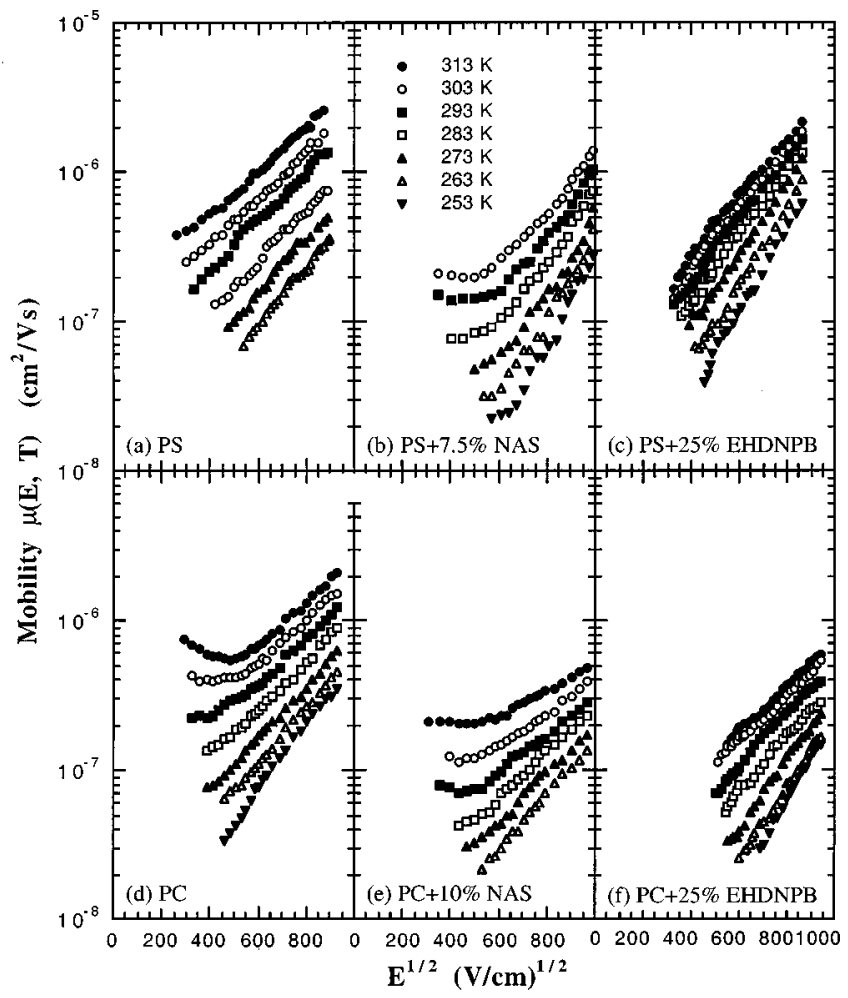

FIG. 4. Logarithm of the mobility vs $\sqrt{E}$ for several compositions containing 30 wt $\%$ DEH combined with: (a) PS, (b) PS $+7.5 \%$ NAS, (c) PS $+25 \%$ EHDNPB, (d) PC, (e) PC $+10 \%$ NAS, (f) PC $+25 \%$ EHDNPB.

The carrier mobility $\mu$ was determined from the conventional expression, $\mu=L^{2} / \tau V$, where $L$ is the thickness of the charge transport layer, $\tau$ is the transit time of the charge carriers, and $V$ is the voltage across the polymer layer. The photocurrent transients mostly show a clear plateau and knee at the transit time $\tau$ [Figs. 3(a) and 3(b)], indicating weak dispersion, in all the systems at most temperatures and applied electric fields. However, at high temperatures and high electric fields, cusps were often observed as shown in Fig. $3(\mathrm{c})$. Therefore, the transit times $\tau(T, E)$ for all systems were determined from the intersection of the asymptotes to the plateau (or cusp) and trailing edge of the transients, in double linear current versus time representation (Fig. 3). The measurements were repeated for a range of applied electric fields and temperatures. The TOF technique and the method of extracting transit times were described in greater detail elsewhere. ${ }^{11,20,39,44}$

\section{RESULTS}

\section{A. Dependence of mobility on electric field and temperature}

The form of Eq. (1) requires that the log of the mobility plotted against $\sqrt{E}$ will be a straight line, the familiar PooleFrenkel form. Figure 4 shows a series of these plots parameterized in temperature for several composites containing 30 wt $\%$ of the hole transport agent DEH. These plots are linear for fields above $160 \mathrm{kV} / \mathrm{cm}$, but some of the plots curve up at 


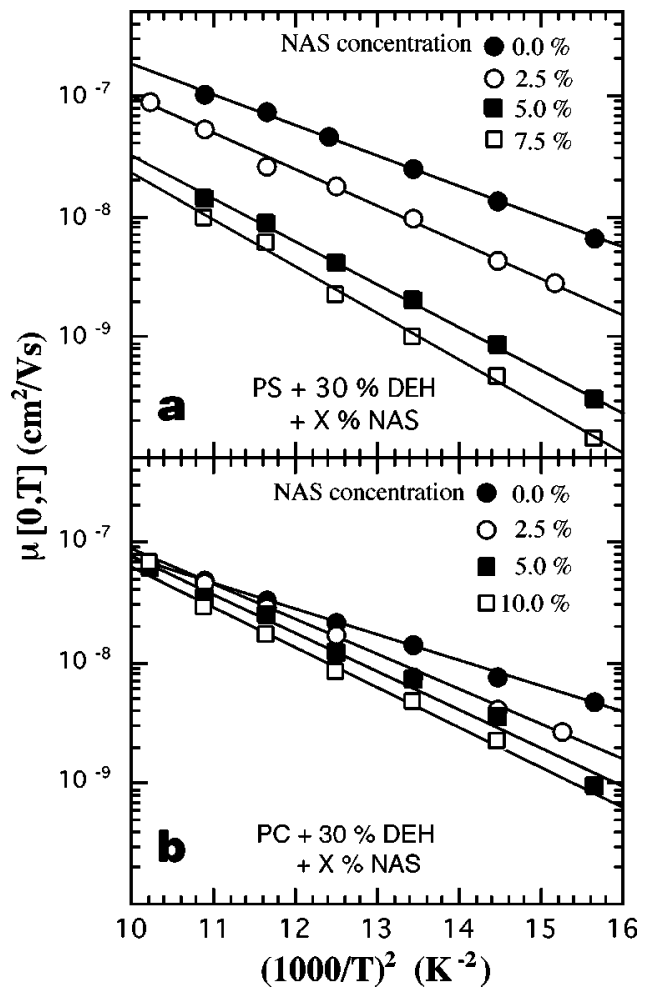

FIG. 5. Logarithm of the zero-field mobility $\mu(E=0)$ vs $T^{-2}$ in samples of host polymer (a) PS and (b) PC containing $30 \mathrm{wt} \%$ DEH and different concentrations by weight of NAS.

low fields and low mobilities, likely due to the dominance of positional disorder in this regime., ${ }^{9,20,21}$ The linear high-field section of each plot in Fig. 4 has the form

$$
\ln \mu(E, T)=\ln \mu(0, T)+\beta(T) \sqrt{E} .
$$

According to Eq. (1), the intercept yields the zero-field mobility $\mu(0, T)=\mu_{0} \exp \left[-\left(2 \sigma / 3 k_{B} T\right)^{2}\right]$ that depends on the energy width $\sigma$, and the slope $\beta(T)=C\left[\left(\sigma / k_{B} T\right)^{2}-\Sigma^{2}\right]$ depends on both the energy width $\sigma$ and the parameter $\Sigma$ representing the degree of positional disorder.

The temperature dependencies of $\mu(0, T)$ and $\beta(T)$ are confirmed by the plots in Figs. 5 and 6, for the narrow temperature range $253-313 \mathrm{~K}$, where the solid lines are leastsquares fits to the functions given following Eq. (4). Figure 5 shows the temperature dependence of the zero-field mobility $\mu(0, T)$ for NAS-doped compositions. In all systems $\mu(0, T)$ decreases with decreasing temperature or increasing chromophore concentration. The slope $\left[\left(2 \sigma / 3 k_{B}\right)^{2}\right]$ of each plot determines the energy width $\sigma$ and the intercept determines the prefactor mobility $\mu_{0}$ for each composition. The temperature dependence of the Poole-Frenkel coefficient $\beta(T)$ $=\partial(\ln \mu) / \partial\left(E^{1 / 2}\right)$ for the NAS-doped PC $+\mathrm{DEH}$ and $\mathrm{PS}+\mathrm{DEH}$ is shown in Fig. 6 . The slopes $\left[C\left(\sigma / k_{B}\right)^{2}\right]$ of the plots in Fig. 6 determine the empirical constant $C$ for each composition in conjunction with the respective value of $\sigma$ obtained from the plots in Fig. 5. The intercept $C \Sigma^{2}$ then determines the positional disorder parameter $\Sigma$. The data in Figs. 4-6 and similar data for other compositions were used to extract the values of the parameters $\mu_{0}, C, \sigma$, and $\Sigma$ as listed in Tables II and III.

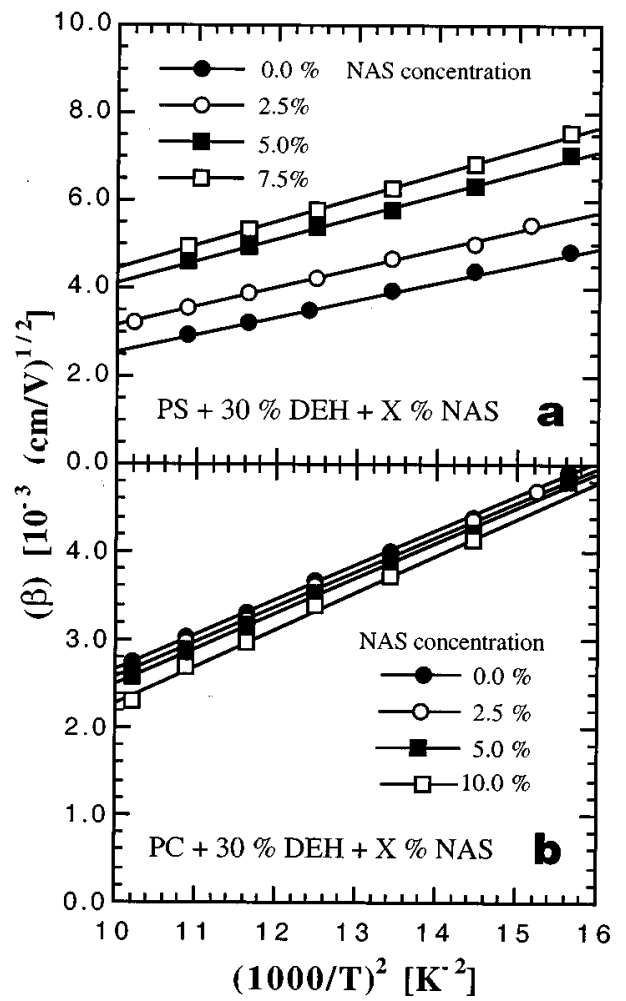

FIG. 6. Poole-Frenkel Coefficient $\beta(T)$ vs $T^{-2}$ in samples of host polymer (a) PS and (b) PC containing $30 \mathrm{wt} \% \mathrm{DEH}$ and different concentrations by weight of NAS.

Figure 4 shows that the mobility in several material compositions follows the Poole-Frenkel $\exp (\beta \sqrt{E})$ form of Eq. (1). As noted above, the data do not distinguish a temperature dependence of the GDM form $1 / T^{2}$ from the Gill form $1 / T$ so that we can calculate an equivalent zero-field activation energy $\Delta_{0}=8 \sigma^{2} / 9 k_{B} \bar{T}$ ranging from 0.26 to 0.54 $\mathrm{eV}$ for the values of $\sigma$ ranging from 0.083 to $0.122 \mathrm{eV}$. The

TABLE II. Transport parameters for $\mathrm{PC}+30 \% \mathrm{DEH}+$ chromophores at different compositions. (a) For the NAS chromophore, (b) For the EHDPNPB chromophore.

(a)

\begin{tabular}{ccccc}
$\begin{array}{c}\text { NAS } \\
(\text { wt } \%)\end{array}$ & $\begin{array}{c}\sigma \\
(\mathrm{eV})\end{array}$ & $\Sigma$ & $\begin{array}{c}\mu_{0} \\
\left(\mathrm{~cm}^{2} / \mathrm{V} \mathrm{s}\right)\end{array}$ & $\begin{array}{c}C \\
10^{-4}(\mathrm{~cm} / \mathrm{V})^{1 / 2}\end{array}$ \\
\hline 0.0 & 0.100 & 2.11 & $1.02 \times 10^{-5}$ & 2.95 \\
2.5 & 0.105 & 2.21 & $4.21 \times 10^{-5}$ & 2.61 \\
5.0 & 0.110 & 2.42 & $7.88 \times 10^{-5}$ & 2.48 \\
7.5 & 0.112 & 2.62 & $9.87 \times 10^{-5}$ & 2.53 \\
10.0 & 0.115 & 3.11 & $1.49 \times 10^{-4}$ & 2.73
\end{tabular}

(b)

\begin{tabular}{ccccc}
$\begin{array}{c}\text { EHDNPB } \\
(\mathrm{wt} \%)\end{array}$ & $\begin{array}{c}\sigma \\
(\mathrm{eV})\end{array}$ & $\Sigma$ & $\mu_{0}\left(\mathrm{~cm}^{2} / \mathrm{V} \mathrm{s}\right)$ & $\begin{array}{c}C \\
10^{-4}(\mathrm{~cm} / \mathrm{V})^{1 / 2}\end{array}$ \\
\hline 0 & 0.100 & 2.11 & $1.02 \times 10^{-5}$ & 2.95 \\
5 & 0.107 & 2.18 & $1.24 \times 10^{-4}$ & 2.76 \\
10 & 0.109 & 2.37 & $1.58 \times 10^{-4}$ & 2.61 \\
15 & 0.115 & 2.47 & $1.92 \times 10^{-4}$ & 2.84 \\
20 & 0.119 & 2.62 & $2.08 \times 10^{-4}$ & 2.84 \\
25 & 0.122 & 3.57 & $3.19 \times 10^{-4}$ & 3.21 \\
\hline \hline
\end{tabular}


TABLE III. Transport parameters for PS $+30 \% \mathrm{DEH}+$ chromophores at different compositions.

\begin{tabular}{ccccc}
\hline \hline \multicolumn{5}{c}{ Table IIIa: for the NAS chromophore } \\
$\begin{array}{c}\text { NAS } \\
(\mathrm{wt} \%)\end{array}$ & $\begin{array}{c}\sigma \\
(\mathrm{eV})\end{array}$ & $\Sigma$ & $\begin{array}{c}\mu_{0} \\
\left(\mathrm{~cm}^{2} / \mathrm{V} \mathrm{s}\right)\end{array}$ & $\begin{array}{c}C \\
\left.10^{-4}(\mathrm{~cm} / \mathrm{V})\right)^{1 / 2}\end{array}$ \\
\hline 0.0 & 0.096 & 2.14 & $4.87 \times 10^{-5}$ & 3.19 \\
2.5 & 0.104 & 1.98 & $6.22 \times 10^{-5}$ & 2.80 \\
5.0 & 0.106 & 1.78 & $1.74 \times 10^{-4}$ & 3.42 \\
7.5 & 0.110 & 1.73 & $3.72 \times 10^{-4}$ & 3.28 \\
\multicolumn{7}{c}{} \\
EHDNPB & Table IIIb: for the EHDNPB chromophore \\
$(\mathrm{wt} \%)$ & $(\mathrm{eV})$ & $\Sigma$ & $\begin{array}{c}\mu_{0} \\
\left(\mathrm{~cm}^{2} / \mathrm{V} \mathrm{s}\right)\end{array}$ & $10^{-4}(\mathrm{~cm} / \mathrm{V})^{1 / 2}$ \\
\hline 0 & 0.096 & 2.14 & $4.87 \times 10^{-5}$ & 3.19 \\
5 & 0.096 & 2.05 & $4.02 \times 10^{-5}$ & 2.75 \\
10 & 0.098 & 1.82 & $4.23 \times 10^{-5}$ & 3.23 \\
15 & 0.103 & 1.78 & $5.08 \times 10^{-5}$ & 3.04 \\
20 & 0.106 & 1.72 & $6.35 \times 10^{-5}$ & 3.09 \\
25 & 0.107 & 1.78 & $8.60 \times 10^{-5}$ & 3.17 \\
\hline \hline
\end{tabular}

Gill form ${ }^{16}$ of the Poole-Frenkel coefficient can be recovered by a similar substitution.

\section{B. Effect of the dipole moment of the polymer binder and of the chromophore concentration}

Since the prototypical photorefractive polymer is based on a polar host polymer (PC), we began by studying the effect of the host polymer on carrier mobility. Figure 4 shows the general trend of decreased mobility with increased concentration of the polar dopants (NAS and EHDNPB). The measured mobilities are a little higher than those of the prototypical photorefractive polymer bisA-NAS:DEH, in which the NAS chromophore is covalently attached to a PC backbone. ${ }^{9,44}$

The plots in Fig. 7 show the hole mobility $\mu(E, T)$ as a function of electric field strength $E$ at temperature $T$ $=303 \mathrm{~K}$, for $\mathrm{PC}+\mathrm{DEH}$ doped with different concentrations of both chromophores, NAS and EHDNPB. Similar plots were obtained with the PS host, where the carrier mobility always decreases with increasing chromophore concentra-

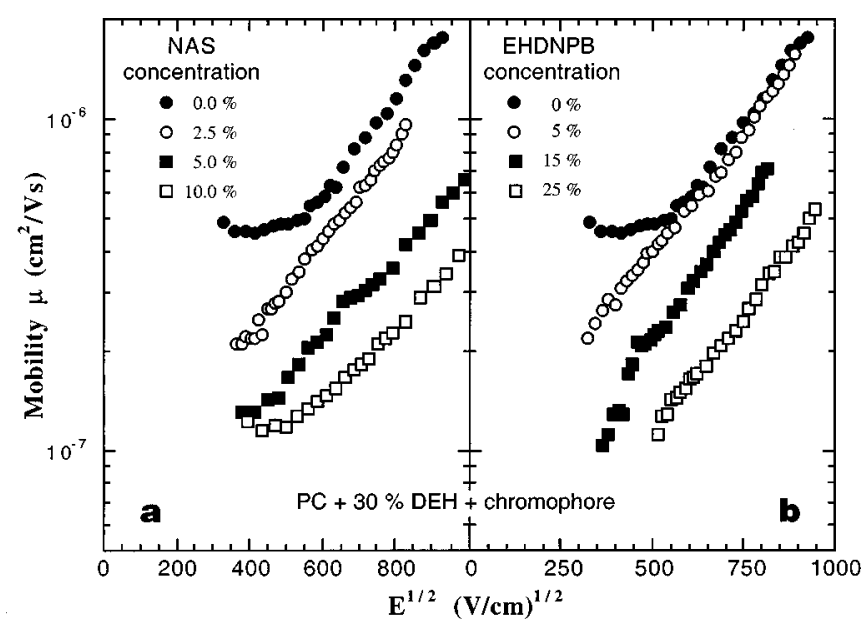

FIG. 7. Logarithm of the mobility vs $\sqrt{E}$ at $303 \mathrm{~K}$. Compositions PC:30\%DEH:X\%chromphore for (a) NAS and (b) EHDNPB chromophores.

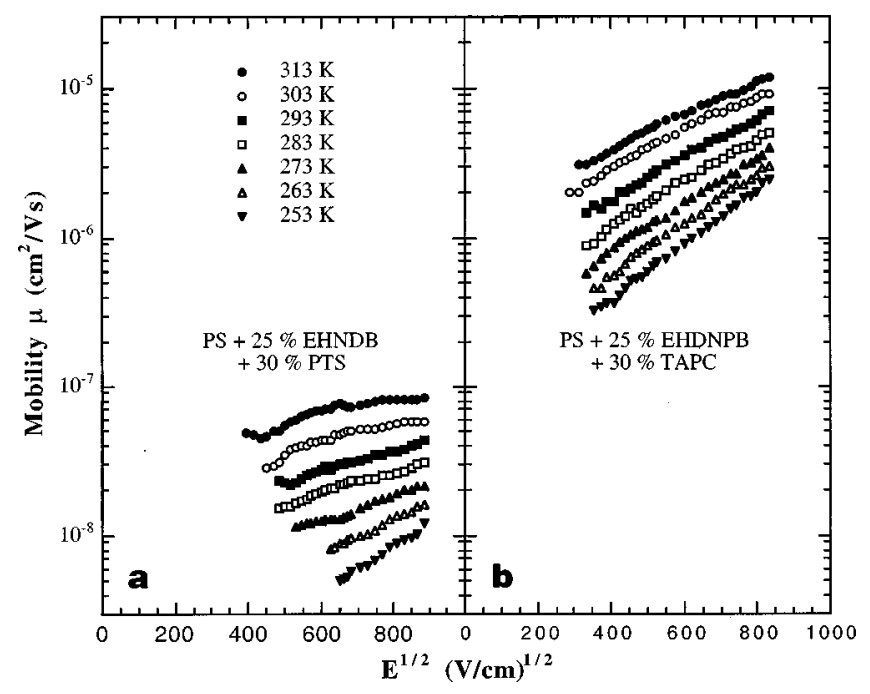

FIG. 8. Mobilities of the composition PS +25 wt $\%$ EHNDPB with (a) 30 wt \% PTS electron transport agent and (b) $30 \mathrm{wt} \%$ TAPC hole transport agent.

tion. (The chromophore concentration was limited by the tendency of the chromophore doped at high concentrations to aggregate and crystallize during or shortly after sample fabrication. ${ }^{47,48}$ )

\section{Effect of dipole moment of the charge transport agent on carrier mobility}

The plots in Fig. 8 show the logarithm of the mobility as a function of $\sqrt{E}$, parametric in temperature, for the compositions containing PS $+25 \mathrm{wt} \% \mathrm{EHDNPB}+30 \mathrm{wt} \%$ of the transport agents with the highest and lowest overall mobilities, hole transport agent TAPC and electron transport agent PTS, respectively. Similar plots were obtained for compositions containing $30 \mathrm{wt} \%$ of four other transport agentsTTA, TAA, DEH, and NTDI-in PS $+25 \mathrm{wt} \%$ EHDNPB. At high fields the mobility follows the form $\exp (\beta \sqrt{E})$ as expected from Eq. (1). As discussed in previous sections, extrapolation of the high field data on a $\ln (\mu)$ versus $\sqrt{E}$ plot [see Eq. (4) and Fig. 4] determines the zero field mobility $\mu(0, T)$ and the slope $\beta(T)$ which were then plotted versus $1 / T^{2}$ (as in Figs. 5-6). The values of the parameters $\sigma, \Sigma, C$, and $\mu_{0}$ extracted from these plots are listed in Table IV. The

TABLE IV. Transport parameters for PS host polymer plus $25 \mathrm{wt} \%$ EHDNPB chromophore plus $30 \mathrm{wt} \%$.

\begin{tabular}{|c|c|c|c|c|c|}
\hline $\begin{array}{c}\text { Transport } \\
\text { agent }\end{array}$ & $\begin{array}{l}\text { Dipole } \\
\text { moment } \\
\text { (D) }\end{array}$ & $\begin{array}{c}\sigma \\
(\mathrm{eV})\end{array}$ & $\Sigma$ & $\begin{array}{c}\mu_{0} \\
\left(\mathrm{~cm}^{2} / \mathrm{V} \mathrm{s}\right)\end{array}$ & $\begin{array}{c}C \\
10^{-4}(\mathrm{~cm} / \mathrm{V})^{1 / 2}\end{array}$ \\
\hline \multicolumn{6}{|c|}{ Hole transport agents } \\
\hline TTA & $0.8(h)$ & 0.0831 & 1.82 & $1.1 \times 10^{-5}$ & 3.1 \\
\hline TAPC & $1.0(h)$ & 0.0966 & 1.97 & $4.1 \times 10^{-4}$ & 2.7 \\
\hline TAA & $2.1(h)$ & 0.1047 & 1.73 & $8.2 \times 10^{-6}$ & 3.3 \\
\hline DEH & $3.2(h)$ & 0.1071 & 1.78 & $8.6 \times 10^{-5}$ & 3.2 \\
\hline \multicolumn{6}{|c|}{ Electron transport agents } \\
\hline NTDI & $3.3(e)$ & 0.1146 & 2.91 & $2.7 \times 10^{-4}$ & 2.6 \\
\hline PTS & $4.0(e)$ & 0.1154 & 3.89 & $1.6 \times 10^{-4}$ & 2.6 \\
\hline
\end{tabular}




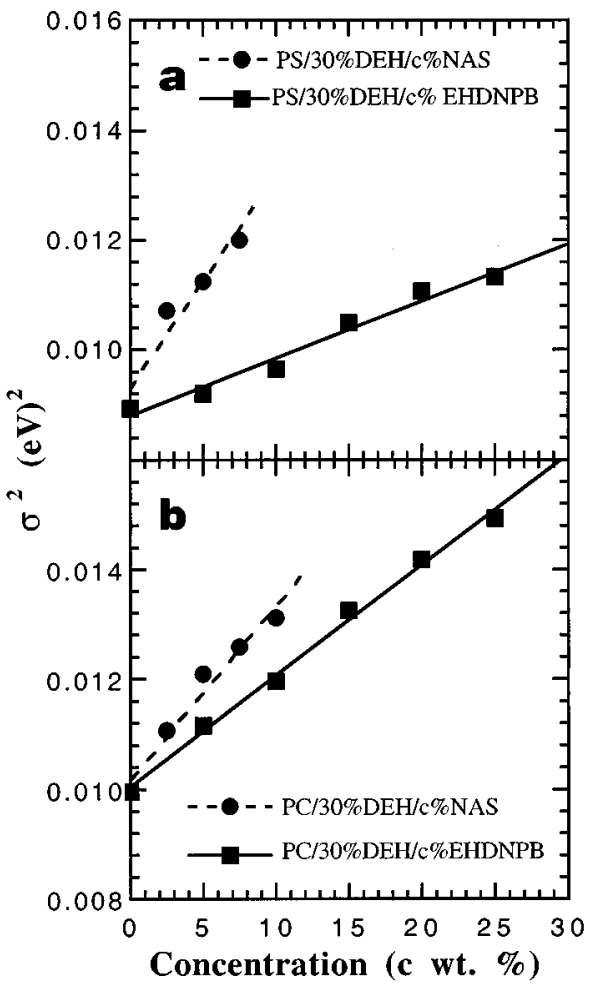

FIG. 9. Increase of the energy width $\sigma$ with concentration $c$ of the electrooptic chromophores for charge transport systems with $30 \mathrm{wt} \% \mathrm{DEH}$ : (a) for PS host and (b) for PC host. The straight lines are least-squares fits to Eq. (5) for exponent $n=1 / 2$

values of $\sigma$ increase systematically with increasing dipole moment of the transport agent while the values of $\Sigma$ and $C$ appear to depend only on the sign of the carrier and $\mu_{0}$ does not show any clear trend.

\section{DISCUSSION}

The mobility data at electric fields above $250 \mathrm{kV} / \mathrm{cm}$ reasonably fit Eq. (1), suggesting that it is reasonable to analyze the data in the framework of the GDM. ${ }^{20,26}$ The data do not cover a wider range of temperatures or fields than previous reports, and therefore are not significantly more stringent tests of the GDM, nor do the results completely rule out other models that approximate the $\exp (\beta \sqrt{E})$ dependence. ${ }^{14,23-25,27,38}$ The purpose of the present study is to test the dipolar disorder models in systems with large concentrations of highly polar chromophores and also to find a means to improve the sensitivity of photorefractive polymers.

The data confirm that dipolar constituents increase the energy width $\sigma$ and thus reduce the mobility, in agreement with previous studies ${ }^{29-32,50}$ and with dipolar disorder model calculations. ${ }^{28,35,38}$ The first confirmation is the lower mobility in the polar PC host versus the weakly polar PS host, as shown in Fig. 4. The second confirmation is the reduction of mobility with the addition of polar dopants (NAS and EHDNPB), as shown Figs. 4, 7, and 9. The third confirmation is dependence of the mobility on the dipole moment of the transport agent itself, as shown in Fig. 10. Note that our studies differ from previous studies on the effect of host

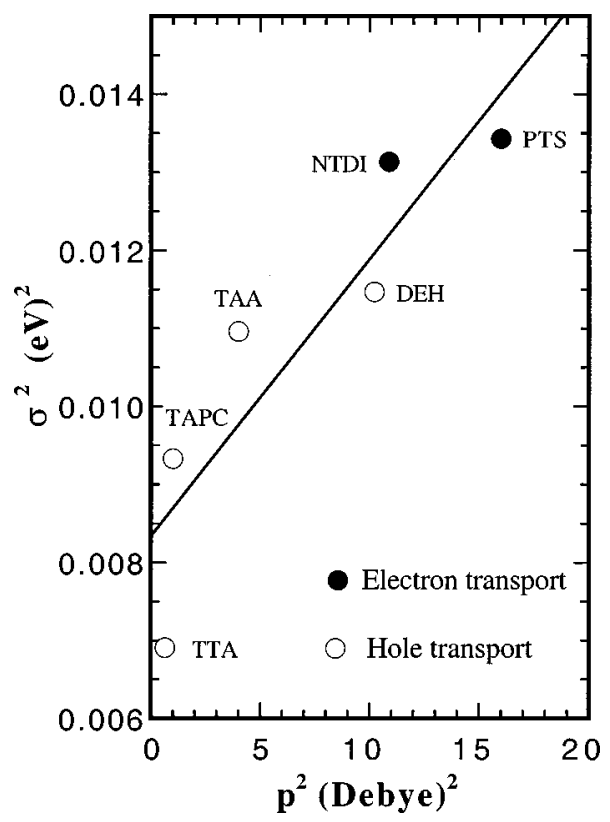

FIG. 10. Increase of the Energy width $\sigma$ with transport agent dipole moment $p$ for charge transport systems with PS +25 wt $\%$ EHNDPB. The straight lines are least-squares fits to Eq. (5).

polymer binder ${ }^{32,50}$ and the effect of the charge transport agent ${ }^{29-31}$ as our systems contain significant concentrations of strongly polar chromophores (NAS and EHDNPB) in addition to polar host and transport agents. The increased width $\sigma$ of the site energy distribution is a consequence of the random electrostatic potentials of the polar molecules. In turn, the reduced mobility is a consequence of the increased energy mismatch between neighboring hopping sites.

The dipolar disorder models predict that the total energy width $\sigma$ includes two independent contributions, a dipolar contribution $\sigma_{d}$ and a van der Waals contribution $\sigma_{\mathrm{vdw}}$, added in quadrature [Eq. (2)]. The dipolar contribution $\sigma_{d}$ is proportional to the dipole moment $p$ and to a power $n$ of the concentration of dipoles $c$ [Eq. (3)], where $n=1 / 2$ or $2 / 3$ depending on the model. Combining Eqs. (2) and (3) yields the dependence of the energy width $\sigma$ on concentration $c$ and dipole moment $p$,

$$
\sigma^{2}=\sigma_{\mathrm{vdw}}^{2}+A^{2} \frac{c^{2 n} p^{2}}{\kappa^{2} a^{2 m}}
$$

Figure 9 shows the dependence of the energy width $\sigma$ on the concentration $c$ of two chromophores, NAS and EHDNPB, in both PC and PS polymer hosts with $30 \mathrm{wt} \% \mathrm{DEH}$. The plots for the four systems test the validity of Eq. (5) for a doped polymer containing a strong dipolar additive in addition to a polar charge transport agent. The data are more consistent with the linear dependence on concentration, $n$ $=1$, proposed by Young ${ }^{35}$ and Dunlap, Parris, and Kenkre, ${ }^{38}$ while the superlinear dependence, $2 n=4 / 3$, of Dieckman, Bässler, and Borsenberger ${ }^{36}$ and Hirao and Nishizawa ${ }^{37}$ would be too strong. The NAS chromophores have a larger effect on the energy width despite having a smaller dipole moment $(6.7 \mathrm{D})$ than EHDNPB (7.6 D), but EHDNPB is much larger because of its alkyl tail [see Fig. 1(b)] and there- 
fore results in a smaller dipole density per unit concentration $c$. The polar chromophores have a much larger effect on the energy width $\sigma$ in the more polar host (PC), suggesting that the host polymer binder and chromophore contributions are not independent. Similar behavior has been observed in other MDPs containing only polar charge transport agents. ${ }^{32,50,51}$

In addition to the energetic disorder, the positional disorder also contributes significantly to reducing the carrier mobility. The two systems, PC:NAS:DEH and PC:EHDNPB:DEH show an increase in the positional disorder parameter $\Sigma$ with increasing chromophore concentration, but in other two systems PS:NAS:DEH and PS:EHDNPB:DEH show a decrease in $\Sigma$ with increasing chromophore concentration (Tables II and III). The decrease in $\Sigma$ with increasing chromophore concentration is a common observation in many molecularly doped polymer systems containing polystyrene (PS) as the host polymer binder doped with a strong polar species, ${ }^{33,41,42}$ and does not agree with the qualitative prediction of the dipolar disorder model that increased concentrations should increase $\Sigma$. Some of the data [see Figs. 4(b), 4(d), and 4(e)] show decreasing mobility with increasing field at low field regime, contrary to the predictions of Eq. (1). This behavior is also seen in the full Monte Carlo simulations of the standard GDM (Ref. 20) and of a polaronhopping variant, ${ }^{24}$ and in measurements on photorefractive polymers $^{9}$ and in other MDPs. ${ }^{20,28,29,40,52,53}$ This behavior is attributed to the large positional disorder $\Sigma$. At low fields, the charge carriers can hop through many percolation paths. But the increasing field reduces the number of percolation paths by inhibiting hops against the applied field, thus reducing the mobility. The random electric fields of the polar molecule would further interrupt the topological connectivity.

The energy width $\sigma$ increases with transport agent dipole moment $p$ in fair agreement with Eq. (5) as shown in Fig. 10. The electron and hole transport agents with dipole moments ranging from 0.8 to $4.0 \mathrm{D}$ (see Table I) were mixed at 30 wt \% with $25 \mathrm{wt} \%$ EHDNPB in the host polymer PS. Table IV summarizes the data used for Fig. 10, and other transport parameters. The results follow the general trend of Eq. (5), an increase in energy width $\sigma$ with dipole moment of the transport agents, consistent with the predictions of dipolar disorder models. However, there is significant scatter about the best fit line and it is most likely due to the differing van der Walls contributions from each transport agent. The average van der Waals contribution $\sigma_{\mathrm{vdw}}=0.093 \mathrm{eV}$ is obtained from the intercept of the least-squares fit line in Fig. 10. This value is comparable with the van der Waals contribution in most MDPs. ${ }^{30,31,40,54,55}$ Similarly, the slope of the fit line in Fig. 10 is $d \sigma^{2} / d p^{2}=0.00041(\mathrm{eV} / \mathrm{D})^{2}$ is comparable to the value $0.00035(\mathrm{eV} / \mathrm{D})^{2}$ obtained with the same transport agents (plus some others) in PS without but without additional chromophores. ${ }^{54,55}$

The overall effect of the dipole moment of the transport agents on the carrier mobility is clearly shown in Fig. 11, where mobility of each system containing PS $+25 \%$ EHDNPB and $30 \mathrm{wt} \%$ of the different transport agents is plotted against $\sqrt{E}$ at $T=303 \mathrm{~K}$. It also shows the combined effect of energetic and spatial disorder, where the

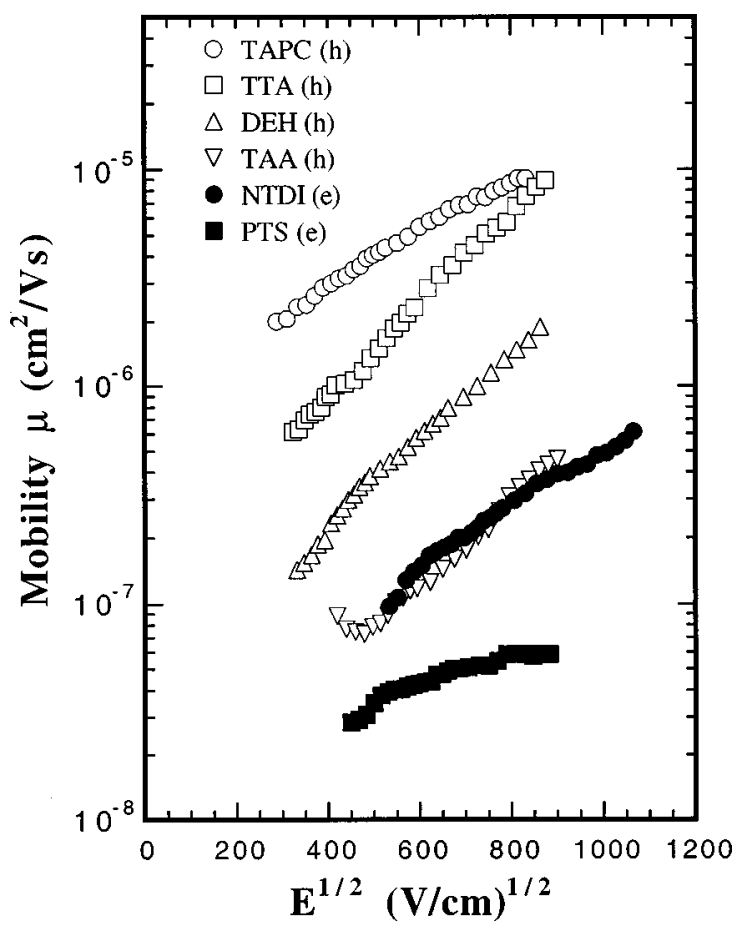

FIG. 11. Field dependence of the mobility at $T=303 \mathrm{~K}$ for $\mathrm{PS}+25 \mathrm{wt} \% \mathrm{EHNDPB}+30 \mathrm{wt} \%$ of the six different charge transport agents.

mobility is reduce dramatically with increasing dipole moment of the transport agent-photorefractive polymers, for example.

\section{CONCLUSIONS}

This study clearly shows that the polar additives play a dominant role in controlling both energetic and spatial disorder and lead to significantly reduced carrier mobility in good quantitative agreement with the dipolar disorder models. ${ }^{35,36,38}$ These are the first results showing the dipolar disorder effects due to independent dopants (NAS and EHDNPB) and the transport agent study reveals that the proper choice of transport agents is important in achieving high mobilities in MDPs that contain other strong polar species.

The results underline a key question: how to optimize both gain and speed in photorefractive polymers? High gain requires polar chromophores to produce the necessary electro-optic response, ${ }^{1}$ or orientational enhancement effect, ${ }^{56}$ yet these same chromophores reduce the carrier mobility and thus degrade the speed. The present study shows that proper choice of transport agents and a host polymer with low dipole moments should improve speed by more than two orders of magnitude. Another approach might be to reduce dipole moment of the chromophore while maintaining the electro-optic response though this has limitations since the electro-optic response is normally proportional to the chromophore dipole moment. In any case, it is essential to continue studying the effects of polar molecules on charge transport in organic photorefractive materials in order to obtain a better understanding of the transport phenomena in this complex system and also to find the other ways to increase response speeds. 


\section{ACKNOWLEDGMENTS}

This work was supported by the Air Force Office of Scientific Research and by the Nebraska Research Initiative through the Center for Materials Research and Analysis. The authors thank Paul Borsenberger, David Dunlap, and Alexander Bune for helpful suggestions and advice, and Mark Meldrim and Toshiyuki Matsui for their help in sample preparation. They also thank Paul Borsenberger and Ed Magin, from Kodak Research laboratories, for the Ni-coated polyethylene terephthalate and the transport agents and for advice on TOF sample preparation.

${ }^{1}$ S. Ducharme, R. W. Twieg, J. C. Scott, and W. E. Moerner, Phys. Rev. Lett. 66, 1846 (1991).

${ }^{2}$ M. Liphardt, A. Goonesekera, B. E. Jones, S. Ducharme, J. M. Takacs, and L. Zhang, Science 263, 367 (1994).

${ }^{3}$ K. Meerholz, B. L. Volodin, Sandalphon, B. Kippelen, and N. Peyghambarian, Nature (London) 371, 497 (1994).

${ }^{4}$ A. Grunnet-Jepson, C. L. Thompson, and W. E. Moerner, Science 277, 549 (1997).

${ }^{5}$ E. Hendrickx, J. Herlocker, J. L. Maldonado, S. R. Marder, B. Kippelen, A. Persoons, and N. Peyghambarian, Appl. Phys. Lett. 72, 1679 (1998).

${ }^{6}$ B. Kippelen, J. Herlocker, J. L. Maldonado, K. Ferrio, E. Hendrickx, S. Mery, A. Golemme, S. R. Marder, and N. Peyghambarian, Proc. SPIE 3471, 22 (1998).

${ }^{7}$ D. Wright, M. A. Diaz-Garcia, J. Casperson, M. S. DeClue, W. E. Moerner, and R. J. Twieg, Appl. Phys. Lett. 73, 1490 (1998).

${ }^{8}$ A. Goonesekera, S. Ducharme, J. M. Takacs, and L. Zhang, Proc. SPIE 3144, 195 (1997).

${ }^{9}$ A. Goonesekera, S. Ducharme, J. M. Takacs, and L. Zhang, J. Chem. Phys. 107, 8709 (1997).

${ }^{10}$ A. Goonesekera, and S. Ducharme, Proc. Opt. Soc. Am. 14, 248 (1997).

${ }^{11}$ A. Goonesekera, S. Ducharme, and J. M. Takacs, Proc. SPIE 3471, 4 (1998).

${ }^{12}$ L. Solymar, D. J. Webb, and A. Grunnet-Jepsen, The Physics and Applications of Photorefractive Materials (Clarendon, Oxford, 1996).

${ }^{13}$ Photorefractive Materials and Their Applications II: Survey of Applications, edited by P. Günter and J.-P. Huignard (Springer, Berlin, 1989), Vol. 62.

${ }^{14}$ P. M. Borsenberger and D. S. Weiss, Organic Photoreceptors for Xerography (Dekker, New York, 1998).

${ }^{15}$ D. M. Pai, J. Chem. Phys. 52, 2285 (1970).

${ }^{16}$ W. D. Gill, J. Appl. Phys. 43, 5033 (1972).

${ }^{17}$ J. X. Mack, L. B. Schein, and A. Peled, Phys. Rev. B 39, 7500 (1989).

${ }^{18}$ D. Emin, Proceedings of the Scottish Universities Summer School in Physics, Thirteenth Session, edited by P. G. LeComber and J. Mort (Academic, Aberdeen, Scotland, 1973), Chap. 7.

${ }^{19}$ A. Miller and E. Abrahams, Phys. Rep. 120, 745 (1960).

${ }^{20}$ P. M. Borsenberger, L. Pautmeier, and H. Bässler, J. Chem. Phys. 94, 5447 (1991).
${ }^{21}$ Y. N. Garstein and E. M. Conwell, Phys. Rev. B 51, 6947 (1995).

${ }^{22}$ H. Scher and E. W. Montroll, Phys. Rev. B 12, 2455 (1975).

${ }^{23}$ L. B. Schein, Philos. Mag. B 65, 795 (1992).

${ }^{24}$ Y. N. Garstein and E. M. Conwell, Chem. Phys. Lett. 217, 41 (1994).

${ }^{25}$ D. H. Dunlap, Phys. Rev. B 52, 939 (1995).

${ }^{26}$ H. Bässler, Phys. Status Solidi B 175, 15 (1993).

${ }^{27}$ Y. N. Garstein and E. M. Conwell, J. Chem. Phys. 100, 9175 (1994).

${ }^{28}$ P. M. Borsenberger and H. Bässler, J. Chem. Phys. 95, 5327 (1991).

${ }^{29}$ P. M. Borsenberger and H. Bässler, Phys. Status Solidi B 170, 291 (1992).

${ }^{30}$ P. M. Borsenberger and M. B. O'Regan, Chem. Phys. 200, 257 (1995).

${ }^{31}$ E. H. Magin, W. T. Gruenbaum, and P. M. Borsenberger, Jpn. J. Appl. Phys., Part 1 35, 3930 (1996).

${ }^{32}$ J. A. Sinicropi, J. R. Cowdery-Corvan, E. H. Magin, and P. M. Borsenberger, Proc. SPIE 2850, 202 (1996).

${ }^{33}$ R. H. Young and J. J. Fitzgerald, J. Chem. Phys. 102, 9380 (1995).

${ }^{34}$ M. Sugiuchi and H. Nishizawa, J. Imaging Sci. Technol. 37, 245 (1993).

${ }^{35}$ R. H. Young, Philos. Mag. B 72, 435 (1995)

${ }^{36}$ A. Dieckmann, H. Bässler, and P. M. Borsenberger, J. Chem. Phys. 99, 8136 (1993)

${ }^{37}$ A. Hirao and H. Nishizawa, Phys. Rev. B 56, R2904 (1997).

${ }^{38}$ D. H. Dunlap, P. E. Parris, and V. M. Kenkre, Phys. Rev. Lett. 77, 542 (1996).

${ }^{39}$ A. Goonesekera, Ph.D. dissertation, University of Nebraska, Lincoln (1998).

${ }^{40}$ P. M. Borsenberger and S. J. Rossi, J. Chem. Phys. 96, 2390 (1992).

${ }^{41}$ L. B. Schein and P. M. Borsenberger, Chem. Phys. 177, 773 (1993).

${ }^{42}$ P. M. Borsenberger and D. S. Weiss, J. Imaging Sci. Technol. 41, 185 (1997).

${ }^{43}$ W. E. Moerner and S. Silence, Chem. Rev. 94, 127 (1994).

${ }^{44}$ A. Goonesekera, S. Ducharme, J. M. Takacs, and L. Zhang, Proc. SPIE 2850, 41 (1996).

${ }^{45}$ S. M. Silence, C. A. Walsh, J. C. Scott, T. J. Matray, R. J. Twieg, F. Hache, G. C. Bjorklund, and W. E. Moerner, Opt. Lett. 17, 1107 (1992).

${ }^{46}$ S. Ducharme, B. Jones, J. M. Takacs, and L. Zhang, Opt. Lett. 18, 152 (1993).

${ }^{47}$ B. E. Jones, S. Ducharme, M. Liphardt, A. Goonesekera, J. M. Takacs, L. Zhang, and R. V. Athalye, J. Opt. Soc. Am. B 11, 1064 (1994).

${ }^{48}$ B. E. Jones, M.S. thesis University of Nebraska, Lincoln (1993).

${ }^{49}$ A. M. Cox, R. D. Blackburn, D. P. West, T. A. King, F. A. Wade, and D. A. Leigh, Appl. Phys. Lett. 68, 2801 (1996).

${ }^{50}$ T. Sasakawa, T. Ikeda, and S. Tazuke, J. Appl. Phys. 65, 2750 (1989).

${ }^{51}$ H.-J. Yuh and D. M. Pai, Philos. Mag. Lett. 62, 61 (1990).

${ }^{52}$ A. Ioannidis, M. F. Lawrence, H. Kassi, R. Coté, J. P. Dodelet, and R. M. Leblanc, Chem. Phys. Lett. 205, 46 (1993)

${ }^{53}$ S. Huen and P. M. Borsenberger, Physica B 216, 43 (1995).

${ }^{54}$ P. M. Borsenberger, W. T. Gruenbaum, and E. H. Magin, Proc. SPIE 2526, 63 (1995).

${ }^{55}$ P. M. Borsenberger and W. T. Gruenbaum, J. Polym. Sci., Part B: Polym. Phys. 34, 575 (1996).

${ }^{56}$ W. E. Moerner, S. M. Silence, F. Hache, and G. C. Bjorklund, J. Opt. Soc. Am. B 11, 320 (1994). 